\title{
TRIVIALITY OF FIBERS FOR MISIUREWICZ PARAMETERS IN THE EXPONENTIAL FAMILY
}

\author{
ANNA MIRIAM BENINI
}

\begin{abstract}
We consider the family of holomorphic maps $e^{z}+c$ and show that fibers of postsingularly finite parameters are trivial. This can be considered as the first and simplest class of non-escaping parameters for which we can obtain results about triviality of fibers in the exponential family.
\end{abstract}

\section{INTRODUCTION}

An entire transcendental function is a function which is holomorphic on $\mathbb{C}$ and has a unique essential singularity at infinity. Any such function is a local covering outside the set of singular values, i.e. the values at which not all branches of the inverse function are defined and univalent: this includes critical values, asymptotic values and any of their accumulation points.

Among transcendental functions, the complex exponential family $f_{c}(z)=e^{z}+c$ is one of the most widely studied examples, because there is only one singular value, namely at $c$, and because in some respects it can be seen as the combinatorial limit of unicritical polynomials of degree $D$ (see e.g. [B et al.]).

As usual, in one-dimensional complex dynamics, if $f: \mathbb{C} \rightarrow \mathbb{C}$ is a holomorphic function, investigation of the dynamics of $f$ starts with the definition of the Fatou set

$$
F(f):=\left\{z \in \mathbb{C}:\left\{f^{n}\right\} \text { is a normal family in a neighborhood of } z\right\}
$$

and the Julia set $J(f)$ as the complement of the Fatou set. We will say that a point $z$ is escaping if $\left|f^{n}(z)\right| \rightarrow \infty$ as $n \rightarrow \infty$; we will say that it is non-escaping otherwise.

The foundational work in the study of the exponential family has been set by Baker and Rippon [BR] and by Eremenko and Lyubich [EL]; among other properties, they prove (for exponential maps and for more general entire functions respectively) that there are no wandering Fatou components for $e^{z}+c$ (see e.g. [EL], Theorem 3). From this and from the classification of periodic Fatou components (see e.g. Theorem in 16.1 in [Mi]) it follows that if there are no attracting orbits, parabolic orbits or Siegel disks, the Fatou set is empty and hence the Julia set equals $\mathbb{C}$.

Received by the editors September 9, 2009 and, in revised form, March 15, 2010, May 17, 2010, September 29, 2010, October 14, 2010, October 19, 2010, and May 5, 2011.

2010 Mathematics Subject Classification. Primary 37F10, 37F20, 37F45.

(C)2011 American Mathematical Society Reverts to public domain 28 years from publication 
We will make use of the combinatorial structure of the dynamical plane and of the parameter plane worked out by Schleicher, Zimmer and Rempe; however, many other aspects have been investigated, among others, by Devaney and coauthors, Karpinska, and Urbanski. For a review of exponential dynamics and a more complete set of references see, for example, $\underline{\mathrm{R}} 0$.

Given a family $\mathcal{F}$ of holomorphic maps we define the set of structurally stable maps in $\mathcal{F}$ as the set of maps $f \in \mathcal{F}$ for which all maps in a sufficiently small neighborhood of $f$ are topologically conjugate to $f$ itself; we denote this set by $S(\mathcal{F})$ or just $S$. By a theorem of Eremenko and Lyubich ([EL], Theorem 10), this set is an open and dense subset of $\left\{f_{c}(z)=e^{z}+c: c \in \mathbb{C}\right\}$. The complement of $S$ is called the bifurcation locus.

Structural stability can be defined in greater generality for subclasses of the class of transcendental functions with only finitely many singular values. In this class it is possible to define natural finite dimensional parameter spaces of functions (EL], Section 3) in which structurally stable parameters are dense (EL, Theorem 10).

In this setting we will call a map hyperbolic if each of its singular values converges to some attracting cycle under iteration. One of the central problems in one dimensional complex dynamics, whenever it can be shown that structural stability is dense in a given parameter space, is to establish whether each structurally stable function is also hyperbolic. This would imply density of hyperbolicity in the corresponding parameter space. Components of $S$ which do not contain hyperbolic parameters are called non-hyperbolic.

The families of functions for which we have the most results about this conjecture are the families of unicritical polynomials of degree $D$, usually parametrized as $P_{c}^{D}(z)=z^{D}+c$. Most proofs of this kind of results involve a construction called the Yoccoz puzzle and estimates on the modulus of the annuli between puzzle pieces; in the exponential family most dynamically arising objects including puzzle pieces are unbounded, so that the corresponding annuli are degenerate at infinity, breaking down the general strategy of the proofs.

Very recent results for families of real transcendental functions, using different methods, can be found in $\mathrm{RvS}$.

A different way of approaching this problem is to study combinatorial properties (see Section 4) of maps which are topologically conjugate. In particular, the structure of periodic dynamic rays landing together (see Section 2 for the definition of rays, and Section 4 for a discussion about rays landing together) is a topological invariant. In the exponential family, dynamic rays are labeled by sequences in $\mathbb{Z}^{\mathbb{N}}$ called addresses (see again, Section 2), which are the analog of angles for polynomial dynamic rays.

In this setting, it is possible to state that for some specific parameters, the structure of periodic dynamic rays landing together (the combinatorics) characterizes them uniquely, so that they cannot be topologically conjugate to any other parameter nor be on the boundary of a non-hyperbolic component of $S$; we usually refer to this kind of results as rigidity results. This leads to the definition of parameter fibers as the set of parameters which all have the same combinatorics (see Section 5). By the theory of parabolic bifurcations from [RS1, for two parameters, the fact of having the same combinatorics is equivalent to the fact of not being separated by any pair of periodic parameter rays landing together. 
This paper investigates the properties of parameter fibers of parameters which are postsingularly finite, i.e. those parameters $c$ for which the orbit of the singular value $c$ is finite under iteration of $f_{c}(z)=e^{z}+c$. These parameters are commonly called Misiurewicz parameters in holomorphic dynamics.

Our main result is the following:

Theorem 1.1. Parameter fibers of Misiurewicz parameters are trivial, i.e. given any Misiurewicz parameter $c_{0}$, for any other parameter $c$ which does not belong to one of the finitely many parameter rays landing at $c_{0}$, there is a pair of parameter rays with periodic addresses which land together at a parabolic parameter and separate $c$ from $c_{0}$.

In Section 4 we also study the relation between the combinatorics of the exponential family and the combinatorics of unicritical polynomials; in particular, we give a characterization of the patterns in which rays can land together for exponentials in relation to the pattern in which dynamics rays can land together for unicritical polynomials (see Section 4 for a definition of orbit portraits and characteristic addresses).

We will devote the first section to a collection of relevant results about existence and landing properties of dynamic and parameter rays for the exponential family. The second section will introduce Misiurewicz parameters and their combinatorial properties, followed by a section on orbit portraits where we will prove some explicit theorems about the correspondence of orbit portraits between exponentials and polynomials. After that we will give a short introduction to fibers and rigidity, and in the last section we will present the statement and the proof of Theorem 1.1.

For the exponential family, we will refer as $\Pi_{P}$ to the parameter plane and as $\Pi_{c}$ to the dynamical plane for the parameter $c$.

We will indicate by $P_{D}$ the family of unicritical polynomials of degree $D$, call $\Pi_{P}^{D}$ their parameter plane and $\Pi_{c}^{D}$ the dynamical plane with the dynamics given by $P_{c}^{D}(z)=z^{D}+c$. As usual, $\mathbb{C}$ is the complex plane, $\hat{\mathbb{C}}$ is the Riemann sphere, $\mathbb{D}$ the open unit disk and $\mathbb{S}^{1}$ is the unit circle.

\section{DYNAMIC RAYS AND PARAMETER RAYS}

This section has the purpose of recollecting some of the relevant results about rays and their landing properties. We will briefly introduce dynamic rays (originally called external rays) and parameter rays for unicritical polynomials first and then for exponential maps; subsequently, we will present some of the properties that we need. We will use the same notation for exponentials and polynomials, in order to make it easier to state some theorems in parallel. In both cases, rays will be labeled by sequences in $\mathbb{Z}^{\mathbb{N}}$, equipped with the left sided shift map $\sigma$ which maps a sequence $s=s_{1} s_{2} s_{3} \ldots$ to the sequence $\sigma s=s_{2} s_{3} \ldots$.

A sequence $s$ is preperiodic if there exist $k, n \in \mathbb{N}$ such that $\sigma^{k+n} s=\sigma^{n} s$. The smallest $n$ with this property is called the period of $s$. If $k$ can be chosen to be equal to 0 , we say that $s$ is periodic; otherwise, we say that $s$ is strictly preperiodic, and we call the minimal $k$ for which the above equation holds the preperiod of $s$.

Dynamic and parameter rays for polynomials are a very classical topic in complex dynamics. Excellent references are [Mi], Chapter 18, for dynamic rays; CG], Chapter 8 and $[\mathrm{PR}]$ for parameter rays. 
Let $P_{c}^{D}(z)=z^{D}+c$ be a unicritical polynomial. The map $P_{c}^{D}$ can be seen as a map from $\hat{\mathbb{C}}$ to $\hat{\mathbb{C}}$ with infinity being a superattracting fixed point. By Böttcher's Theorem, there is a holomorphic function $B_{c}$, tangent to the identity at infinity, conjugating the dynamics of $P_{c}^{D}(z)$ in a neighborhood of $\infty$ to the dynamics of $P_{0}^{D}$ outside a closed disk or radius $r>1$ centered at 0 . Its inverse $B_{c}^{-1}$ can be extended as a conjugacy to a maximal open disk $\Delta$ centered at infinity; if $c$ is non-escaping, $\Delta=\hat{\mathbb{C}} \backslash \overline{\mathbb{D}}$, while if $c$ is escaping, $\Delta$ is such that $B_{c}^{-1}(\Delta)$ contains $c$ (see [Mi], Theorem 9.3); so we can assume that $B_{c}$ is defined on $B_{c}^{-1}(\Delta)$, and that in the escaping case $c=B_{c}^{-1}\left(e^{t_{c}} e^{2 \pi i s_{c}}\right)$ for some $t_{c}, s_{c}$.

For $s \in \mathbb{S}^{1}$, call $R_{s}:=\left\{z \in \mathbb{C}: z=e^{t} e^{2 \pi i s}, t>0\right\}$ the straight ray of angle $s$ outside the unit disk, and let $s_{c} \in \mathbb{S}^{1}$ be the angle such that $B_{c}(c) \in R_{s_{c}}$. If $s$ is written in $D$-adic expansion, it can be seen as an element of $\mathbb{Z}^{\mathbb{N}}$, and because $B_{c}$ is a conjugacy, the dynamics of $P_{0}^{D}$ carries $R_{s}$ to $R_{\sigma s}$. When $c$ is non-escaping, given a straight ray $R_{s}$, the set

$$
g_{s}^{c}:=B_{c}^{-1}\left(R_{s}\right)
$$

is a well-defined curve and is called the dynamic ray of angle $s$ (for the polynomial $P_{c}^{D}$ ). It can be parameterized by the parameter $t$, called potential, so that

$$
P_{c}^{D}\left(g_{s}^{c}\right)(t)=g_{\sigma s}^{c}(D t)
$$

in this way, $g_{s}^{c}(t)=B_{c}^{-1}\left(e^{t} e^{2 \pi i s}\right)$.

When $c$ is escaping, $B_{c}^{-1}$ is initially defined only on the disk $\Delta$, and so dynamic rays would be only defined for potential $t>t_{c}$. However, $B_{c}^{-1}$ can be extended further using the functional equation $B_{c}\left(P_{c}^{D}(z)\right)=\left(B_{c}(z)\right)^{D}$, and taking some care when selecting branches of the $D$-th root in order to preserve continuity. In this way, $B_{c}^{-1}$ can be extended to any straight ray $R_{s}$ such that $\sigma^{k}(s) \neq s_{c}$ for any $k \in \mathbb{N}$, and to any ray $R_{s}$ such that $\sigma^{k}(s) \neq s_{c}$ for some $k \in \mathbb{N}$ for any potential $t>t_{k}=t_{c} / D^{k}$. Dynamic rays can then be defined as in the non-escaping case, with the difference that the rays whose angles are preimages of the critical angle $s_{c}$ are broken (in the sense that they are not defined for every potential $t>0$ ).

For $s \in \mathbb{S}^{1}$, the set $G_{s}:=\left\{c \in \mathbb{C}, c \in g_{s}^{c}\right\}$ can be shown to be a simple curve and is called the parameter ray of angle $s$.

Now let us define dynamic and parameter rays for the exponential family. We will use throughout the paper the concept of itinerary with respect to a partition:

Definition 2.1. Let $f: \mathbb{C} \rightarrow \mathbb{C}$ be a function, and $\mathcal{M}=\left\{M_{a_{i}}\right\}_{a_{i} \in \mathcal{A}}$ be a countable collection of pairwise disconnected domains of $\mathbb{C}$ such that each domain is labeled uniquely by a letter in a countable alphabet $\mathcal{A}$. If $f^{j}(z) \in \bigcup_{a_{i} \in \mathcal{A}} M_{a_{i}}$ for all $j \in$ $\mathbb{N} \cup\{0\}$, then we say that the itinerary of $z$ (with respect to $\mathcal{M}$ ) is the sequence $a=a_{1} \ldots a_{n} \ldots$ defined by $f^{j}(z) \in M_{a_{j}}$.

Dynamic rays for the exponential family ([DK] and [SZ2]) have been introduced in analogy with the polynomial case in order to construct symbolic dynamics on the set of escaping points

$$
I\left(f_{c}\right):=\left\{z \in \mathbb{C}:\left|f_{c}^{n}(z)\right| \rightarrow \infty \text { as } n \rightarrow \infty\right\} \subset \Pi_{c} .
$$

Let

$$
S_{j}:=\{z \in \mathbb{C}:(2 j-1) \pi<\operatorname{Im} z<(2 j+1) \pi\},
$$


and consider itineraries of points with respect to this partition, i.e.

$$
\operatorname{itin}(z)=s_{1} s_{2} \ldots \text { if and only if } f^{j}(z) \in S_{s_{j}}
$$

for points whose iterates never belong to the boundary of the partition.

For any such point $z$ we will refer to its itinerary with respect to the strips $S_{j}$ as the address 1 of $z$.

Using the described construction, addresses of points cannot have entries growing faster than iterates of the exponential function. This leads to the following notion.

Definition 2.2. A sequence $s=s_{1} s_{2} \ldots$ is called exponentially bounded if there exists $x \in \mathbb{R}$ such that $\left|2 \pi s_{j}\right|<F^{j}(x)$ for all $j \in \mathbb{N}$, where $F: \mathbb{R} \mapsto \mathbb{R}$ is the growth function $F: t \mapsto e^{t}-1$.

This growth condition turns out to be not only necessary, but also sufficient SZ1, so that all sequences $s$ contained in the set

$$
\mathcal{S}:=\left\{s \in \mathbb{Z}^{\mathbb{N}}: s \text { is exponentially bounded }\right\}
$$

are realized as itineraries of some point $z$.

The set $\mathcal{S}$ has a natural order induced by the usual order relation on the space of sequences over an ordered set.

If $s=s_{1} s_{2} \ldots$, we will say that $|s|=\sup _{i}\left|s_{i}\right|$.

Given an external address $s=s_{1} s_{2} \ldots$ we will define its minimal potential to be

$$
t_{s}:=\inf \left\{t>0: \lim \sup _{k \geq 1} \frac{\left|s_{k}\right|}{F^{k}(t)}=0\right\} \text {. }
$$

Definition, existence and properties of dynamic rays for the exponential family are summarized in the following theorem ([SZ1], Proposition 3.2 and Theorem 4.2):

Theorem 2.3 (Existence of dynamic rays). Let $c$ be a parameter such that $\left|f_{c}^{n}(c)\right|$ does not tend to infinity as $n \rightarrow \infty$. Then, for any $s \in \mathcal{S}$, there exists a unique injective curve $g_{s}^{c}:\left(t_{s}, \infty\right) \rightarrow \mathbb{C}$ consisting of escaping points such that:

- $g_{s}^{c}(t)$ has address $s$ for sufficiently large $t$;

- $f_{c}\left(g_{s}^{c}(t)\right)=g_{\sigma s}^{c}(F(t))$;

- $g_{s}^{c}(t)=2 \pi i s_{1}+t+o\left(e^{-t}\right)$ as $t \rightarrow \infty$.

Given $s \in \mathcal{S}$, we will call the unique curve $g_{s}^{c}$ given by Theorem 2.3 the dynamic ray of address $s$.

As in the polynomial case, if $c$ is escaping, then $c$ belongs to a ray of address $s_{c}$ which cannot be extended to its full potential. The same is true for all the rays which are preimages of the ray $g_{s_{c}}^{c}$; as in the polynomial case, those rays are called broken.

We say that a dynamic ray $g_{s}^{c}$ is periodic, preperiodic or strictly preperiodic if $s$ is a periodic, preperiodic or strictly preperiodic sequence. By uniqueness of the curve $g_{s}^{c}$ given by Theorem 2.3, a ray is periodic if and only if $f_{c}^{m}\left(g_{s}^{c}\right) \subset g_{s}^{c}$ for some $m \in \mathbb{N}$, preperiodic if and only if $f_{c}^{m+k}\left(g_{s}^{c}\right) \subset f_{c}^{k}\left(g_{s}^{c}\right)$ for $m, k>0$, and strictly preperiodic if in addition $k>1$.

The question whether periodic dynamic rays land for the exponential family remained open for some time, and was finally solved by Rempe using the previously

\footnotetext{
${ }^{1}$ This is often referred to as the external address to distinguish it from other kinds of addresses. However, this is the only kind of address we deal with in this paper, so the term address will not create ambiguity.
} 
known fact that periodic rays land for hyperbolic parameters and an argument about persistence of landing inside wakes. This led to the following theorem ( $\mathbb{R} 1$, Theorem 1).

Theorem 2.4 (Landing theorem for periodic dynamic rays). Let $c$ be such that $\left|f_{c}^{n}(c)\right|$ does not tend to infinity as $n \rightarrow \infty$. Then every periodic dynamic ray $g_{s}^{c}$ lands at a repelling or parabolic periodic point; also, every strictly preperiodic dynamic ray lands at a strictly preperiodic point. If $c$ is escaping, the same is true unless $c$ belongs to a ray with preperiodic address, in which case the ray itself and all its preimages are broken.

The construction of parameter rays is also done keeping in mind the fundamental property that parameter rays have for polynomials: a point $c$ belongs to some parameter ray $G_{s}$ in $\Pi_{P}$ if and only if $c$ belongs to the dynamic ray $g_{s}^{c}$ in $\Pi_{c}$. Parameter rays where first introduced in B $\mathrm{B}$ et al. and then classified in full generality by Förster and Schleicher. Their results are summarized in the following theorem ([FS, Theorem 3.7):

Theorem 2.5 (Existence of parameter rays). Let $s \in \mathcal{S}$. Then there is a unique injective curve $G_{s}:\left(t_{s}, \infty\right) \rightarrow \mathbb{C}$, such that, for all $t>t_{s}, c=G_{s}(t)$ if and only if $c=g_{s}^{c}(t)$. Also, $\left|G_{s}(t)-\left(t+2 \pi i s_{1}\right)\right| \rightarrow 0$ as $t \rightarrow \infty$.

Given an address $s \in \mathcal{S}$, we will call the unique curve $G_{s}$ given by Theorem 2.5 the parameter ray of address $s$.

Not much is known about the landing properties of parameter rays. However, it is known ([S0]) that parameter rays of periodic and strictly preperiodic addresses land at parabolic and Misiurewicz parameters respectively.

Combinatorial spaces and cyclic order. Theorems 2.3 and 2.5 establish a correspondence between the set of dynamic and parameter rays and the set of exponentially bounded addresses $\mathcal{S}$ for the exponential family. Moreover, the equation $f_{c}\left(g_{s}^{c}(t)\right)=g_{\sigma s}^{c}(F(t))$ in Theorem 2.3 tells us that the dynamics of an exponential function $f_{c}$ on its set of escaping points $I\left(f_{c}\right)$ is conjugate to the dynamics of the left-sided shift map $\sigma$ on $\mathcal{S}$.

The asymptotic estimates in Theorems 2.3 and 2.5 show that dynamic and parameter rays have a well-defined vertical order at infinity, and that this order coincides with the order of their addresses in $\mathcal{S}$. This is the analog of the cyclic order at infinity for polynomial rays.

For all these reasons we will refer to $\mathcal{S}$ as the combinatorial space for the family $e^{z}+c$.

For the family of unicritical polynomials $P_{D}$, the dynamic and parameter rays are in correspondence with the sequences over $D$ symbols (angles in D-adic expansion), that we can represent as

$$
\mathcal{S}_{D}=\left\{\frac{-D+1}{2}, \ldots, 0, \ldots, \frac{D-1}{2}\right\}^{\mathbb{N}} \quad \text { for } D \text { odd }
$$

and

$$
\mathcal{S}_{D}=\left\{\frac{-D+2}{2}, \ldots, 0, \ldots, \frac{D}{2}\right\}^{\mathbb{N}} \quad \text { for } D \text { even }
$$


As for the exponential family, the dynamics of a unicritical polynomial of degree $D$ on the set of dynamic rays is conjugate to the dynamics of the shift map $\sigma$ on $\mathcal{S}_{D}$; also, dynamic and parameter rays have a cyclic order at infinity which corresponds to the cyclic order on $\mathcal{S}_{D}$ if we identify the sequences modulo $D$.

If $l, s \in \mathbb{Z}^{\mathbb{N}}$ are two sequences, $l=l_{1} l_{2} \ldots$ and $s=s_{1} s_{2} \ldots$, we define the distance

$$
\operatorname{dist}(l, s)=\sum_{s_{k} \neq l_{k}} \frac{1}{2^{k}}
$$

which turns $\mathcal{S}$ and $\mathcal{S}_{D}$ into metric spaces.

The space $\mathcal{S}_{D}$ embeds naturally in $\mathcal{S}$ via the identity map; similarly, if $A \subset \mathcal{S}$ is such that $|s|<N$ for each $s \in A$, then $A$ embeds in $\mathcal{S}_{D}$ via the identity map for each $D>2 N+2$.

We will refer to $\mathcal{S}_{D}$ as the combinatorial space for the family $P_{D}$.

We will refer to this description as combinatorial correspondence between the exponential family and unicritical polynomials of sufficiently high degree $D$.

\section{Misiurewicz PARAMETERS}

Given the exponential family $f_{c}(z)=e^{z}+c$, or a family of unicritical polynomials $P_{c}^{D}(z)=z^{D}+c$, we call a parameter $c_{0}$ Misiurewicz (or postsingularly finite) if the orbit of the unique singular or critical value is finite, hence preperiodic. We will say that an exponential or polynomial map $f_{c_{0}}$ is Misiurewicz (or postsingularly finite) if $c_{0}$ is a Misiurewicz parameter.

In the exponential family, the singular value is an omitted value and hence the postsingular orbit cannot be periodic, so for an exponential map being postsingularly finite is equivalent to say that the singular value $c_{0}$ lands on an orbit $\left\{z_{i}\right\}$ of period $m$ after $k$ iterations, for some integers $k>1, m \geq 1$.

Recall that for holomorphic functions, the boundary of any Siegel disk (or cycle of Siegel disks) is contained in the accumulation set for the orbit of a singular value, and that the immediate basin of any hyperbolic or parabolic orbit contains a singular value (see e.g. Theorems 8.6, 10.15 and 11.17 in Mi]; the proofs are local and hold also in the transcendental setting, see e.g. [Be]). This implies that if for an exponential map, the unique singular value is preperiodic, there cannot be attracting or parabolic basins, nor Siegel disks; hence, by the classification of Fatou components mentioned in the introduction, the Julia set equals $\mathbb{C}$, and the periodic orbit $\left\{z_{i}\right\}$ on which the singular value lands is repelling. We will refer to $\left\{z_{i}\right\}$ as the postsingular periodic orbit.

From the discussion above and the discreteness of solutions of the equation $f_{c}^{k+m}(c)=f_{c}^{k}(c)$ it follows immediately that Misiurewicz parameters belong to the bifurcation locus.

There are a few reasons why proving triviality of fibers (see Section 5 for the definition of fibers and a discussion on rigidity) for Misiurewicz parameters is easier than for other cases. Among them there is a correspondence between dynamical and parameter plane at Misiurewicz parameters. For polynomials, this is a wellknown result (see e.g. [DH], Exposé VIII, Chapter III, Theorem 2, for the quadratic case; the unicritical case can be worked out analogously).

Theorem 3.1. Let $P_{c}^{D}$ be a unicritical polynomial with $c$ Misiurewicz. Then there is at least one and at most finitely many dynamic rays landing at $c$ in $\Pi_{c}^{D}$, and the angle of any of those rays is strictly preperiodic. Moreover, any parameter ray with 
the same angle as one of the rays landing at $c$ in the dynamical plane has to land at c in $\Pi_{P}^{D}$.

The analog of Theorem 3.1 for the exponential family is proven in [SZ1]:

Theorem 3.2 (Correspondence between dynamical and parameter plane). An exponential Misiurewicz parameter $c_{0}$ is the landing point of at least one and at most finitely many parameter rays, say $G_{s_{1}}, \ldots, G_{s_{q}}$, and their addresses $s_{1}<\ldots<s_{q}$ are strictly preperiodic with period $m q$ and preperiod $k$. Moreover, the dynamic rays $g_{s_{1}}, \ldots, g_{s_{q}}$ with the corresponding addresses land at $c_{0}$ in $\Pi_{c_{0}}$.

Together with the fact that parameter rays with strictly preperiodic addresses land [S0, Theorem 3.2 gives a combinatorial classification of postsingularly finite exponential maps; see ([LSV], Theorem 2.6).

Theorem 3.3 (Classification of Misiurewicz exponential maps). For every strictly preperiodic address $s$, there is a unique postsingularly finite exponential map such that the dynamic ray of address s lands at the singular value. Every postsingularly finite exponential map is associated in this way to a positive finite number of strictly preperiodic addresses.

For unicritical polynomials, it is well known that parameter rays with strictly preperiodic angles land at Misiurewicz parameters ([PR]), so Theorem 3.3 offers a natural correspondence between exponential Misiurewicz parameters and polynomial Misiurewicz parameters through the angles/addresses of the parameter rays landing at them.
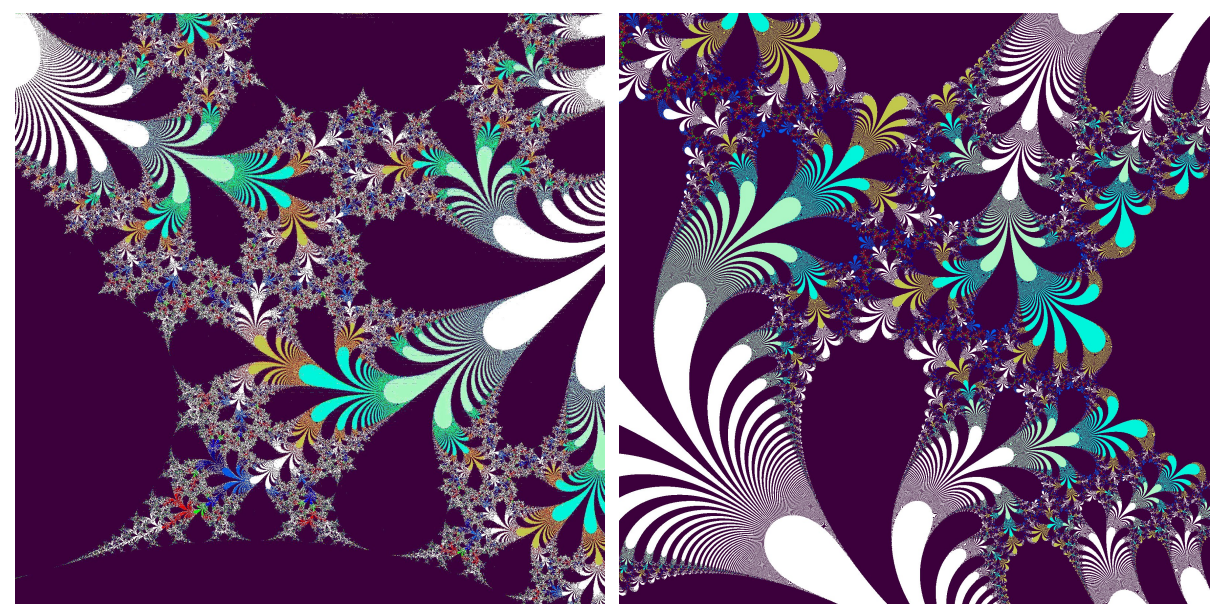

Figure 1. Parameter (on the left) and dynamical plane (on the right) near the exponential Misiurewicz parameter $1.81507+4.70945 \mathrm{i}$; the spiraling of the two rays landing at it can be inferred from the picture.

A combinatorial property of Misiurewicz parameters. One of the features of Misiurewicz parameters that we are going to use in the proof of our main theorem is a lemma connecting topology to combinatorics. It is proved in [SZ1] for exponentials 
and is probably known for unicritical polynomials of degree $D$; for completeness we will include a proof following the outline of [SZ1]. Before stating and proving the lemma, we need to introduce a dynamical partition for Misiurewicz parameters.

Dynamical partition. Let $f(z)=e^{z}+c_{0}$ or $f(z)=z^{D}+c_{0}$ where $c_{0}$ is a Misiurewicz parameter for the map under consideration, and let $g_{s_{1}}$ be one of the finitely many dynamic rays landing at $c_{0}$ given by Theorem 3.2 for exponentials and by Theorem 3.1 for polynomials. The preimage of $g_{s_{1}}$ under $f$ is a set of countably many curves tending to $-\infty$ in the case of exponentials, and a set of $D$ curves connecting at 0 for a polynomial of degree $D$. In both cases, the preimages of $g_{s_{1}}$ partition the plane into domains $W_{j}$.

Similarly, the preimages of $s_{1}$ under the shift map partition the combinatorial spaces $\mathcal{S}$ and $\mathcal{S}_{D}$ into infinitely many sectors for exponentials and $D$ sectors for polynomials. Label with the entry 0 the dynamical and the combinatorial sector containing $c_{0}$ and $s_{1}$ respectively, and label all other sectors using consecutive integers respecting the cyclic/vertical order at infinity.

Any non-escaping point, as well as any ray $g_{s}$ which does not belong to the backward orbit of the ray $g_{s_{1}}$, has a well-defined itinerary whose entries keep track of the sectors visited by the iterates of $s$ under the shift map.

For any dynamic ray $g_{s_{i}}$ landing at the singular value, we call the partition induced by $g_{s_{i}}$ a dynamical partition for $f$.

Different choices of the dynamic ray landing at $c_{0}$ will induce different dynamical partitions; however, this choice will not matter to us.

For the sequel we will need to consider $\mathbb{C} \backslash \bigcup_{n \geq-1} f^{n} g_{s_{1}}$. This gives a new partition of the plane into domains $W_{i, j}$ where, for each fixed $i, W_{i, j}$ denotes a connected component of $W_{i}$.

For convenience of the reader, let us recall that any domain $S$ of $\mathbb{C}$ whose complement contains at least two points, has for universal covering the unit disk. The standard hyperbolic metric of the unit disk can be pushed forward via a universal covering map to obtain a well-defined hyperbolic metric on $S$ whose density we denote by $\rho_{S}$ (for details, see e.g. Chapter 2 in $\underline{\text { Mi }}$ ).

We will use the following basic theorem about hyperbolic contraction ( $\mathrm{Mi}$, Theorem 2.11):

Theorem 3.4 (Schwarz-Pick Lemma). If $f: \Omega \rightarrow \Omega^{\prime}$ is a holomorphic map between two domains admitting a hyperbolic metric, either $f$ is a local isometry, or $f$ strictly decreases all non-zero distances in the hyperbolic metrics of $\Omega$ and $\Omega^{\prime}$, respectively.

A corollary is the monotonicity of the hyperbolic metric:

Lemma 3.5. If $\Omega, \Omega^{\prime}$ are domains admitting a hyperbolic metric with density $\rho_{\Omega}, \rho_{\Omega^{\prime}}$ respectively and $\Omega \subsetneq \Omega^{\prime}$, then $\rho_{\Omega^{\prime}}(z)<\rho_{\Omega}(z)$ for all $z \in \Omega$.

We are now ready to prove the required lemma. For exponentials this lemma appears already in SZ2. We include the proof here, slightly modified, to also cover the case of unicritical polynomials.

Lemma 3.6 (Significance of dynamical partition for Misiurewicz parameters). Let $f=f_{c}$ or $f=P_{c}^{D}$ be a Misiurewicz map. Then two preperiodic dynamic rays which are not preimages of the dynamic rays landing at the singular value land together if and only if they have the same itinerary with respect to the dynamical partition induced by any of the dynamic rays landing at $c$. 
Proof. Let $g_{s_{1}}$ be one of the dynamic rays landing at the singular value and denote by $W_{i}$ the domains obtained when constructing the dynamical partition induced by $g_{s_{1}}$. Endow each of the domains $W_{i}$ and $W_{i, j}$ with the corresponding hyperbolic metric. For any domain $W_{i}$, let $f_{i}^{-1}$ be the inverse branch of $f$ mapping $\mathbb{C} \backslash g_{s_{1}}$ into $W_{i}$. By Theorem 3.4, for any $i, i^{\prime}$ the map $\left.f_{i^{\prime}}^{-1}\right|_{W_{i}}$ contracts the hyperbolic metric from $W_{i}$ to $W_{i^{\prime}}$. By monotonicity of the hyperbolic metric, the hyperbolic metric of each $W_{i, j}$ is bigger than the hyperbolic metric of $W_{i}$, so $\left.f_{i^{\prime}}^{-1}\right|_{W_{i, j}}$ contracts the hyperbolic metric from $W_{i, j}$ to $W_{i^{\prime}}$.

Let us start by considering any two periodic dynamic rays which have the same itinerary with respect to the dynamic partition described in the previous section, and let $w_{1}, w_{2}$ be their periodic landing points. Then $w_{1}$ and $w_{2}$ have the same itinerary, say $\overline{a_{1} \ldots a_{M}}$, and hence are fixed points of the map $\Psi:=f_{a_{M}}^{-1} \circ \cdots \circ f_{a_{1}}^{-1}$.

If for at least one $j, 1 \leq j \leq M$, the $j$-th inverse iterate of $w_{1}$ and $w_{2}$ belong to the same $W_{a_{j}, i}$, and $\operatorname{dist}\left(w_{1}, w_{2}\right) \neq 0$, we have that $\operatorname{dist}\left(\Psi\left(w_{1}\right), \Psi\left(w_{2}\right)\right)<\operatorname{dist}\left(w_{1}, w_{2}\right)$, which is a contradiction as $\Psi\left(w_{1}\right)=w_{1}$ and $\Psi\left(w_{2}\right)=w_{2}$.

This proves the theorem for periodic rays unless iterates of $w_{1}, w_{2}$ always belong to different components of $W_{a_{j}}$. We will show that in this case $w_{1}, w_{2}$ have the same itinerary as a point of the postsingular periodic orbit and that this cannot happen. As $w_{1}, w_{2}$ belong to different $W_{a_{1}, i}$, there are two dynamic rays landing at a postsingular periodic point $z$ which separate $w_{1}$ from $w_{2}$. So one of them, say $w_{1}$, has to belong to one of the sectors bounded by the dynamic rays landing at $z$ which does not contain $-\infty$. This implies that the first entry in the itinerary of $w_{1}$, and hence in the itinerary of $w_{2}$ because the two itineraries are equal, coincides with the first entry in the itinerary of $z$. Using the fact that $f^{j}\left(w_{1}\right), f^{j}\left(w_{2}\right)$ belong to different $W_{a_{j}, i}$ for any $j$, the same reasoning can be repeated to show that $f^{j}\left(w_{1}\right), f^{j}\left(w_{2}\right)$ belong to the same $S_{a_{j}}$ as $f^{j}(z)$, hence that $w_{1}$ and $w_{2}$ have the same itinerary as $z$.

It remains to prove that no periodic point $w$ can have the same itinerary as a postsingular periodic point $z$. Suppose by contradiction that this is the case and let $W_{i, j}$ be the domain such that $z, w \in \overline{W_{i, j}}$. As $z$ and $w$ have the same itinerary, we can find an inverse branch $\Phi$ of $f^{-k}$ fixing both $z$ and $w$. As $W_{i, j}$ does not intersect the postsingular set, $\Phi$ is well defined in all of $W_{i, j}$. If $L$ is a linearizing neighborhood of $z$, as $\Phi$ fixes $z, \Phi^{n} \rightarrow\{z\}$ on $W_{i, j} \cap L$, hence $\Phi^{n} \rightarrow\{z\}$ in $W_{i, j}$ by the identity principle, contradicting the fact that $\Phi(w)=w$.

Now let us consider strictly preperiodic rays. If two strictly preperiodic rays have the same itinerary, their periodic images also have the same itinerary, hence land together by the previous part; and since the strictly preperiodic rays are not preimages of the rays landing at the singular value, and they have the same itinerary, we can take preimages using the same branch for both, so that they keep landing together.

On the other side if two rays land together they form a connected set, which never intersects the original partition under iterates of $f$, so they always belong to the same domain of the partition.

\section{Combinatorics And Ray portraits}

In this section we study the relation between the combinatorics of the exponential family and the combinatorics of unicritical polynomials; in particular, we give a characterization of the patterns in which rays can land together for exponentials 
in relation to the pattern in which dynamic rays can land together for unicritical polynomials. We first introduce orbit portraits (in analogy with [Mi1 for polynomials and RS1] for exponentials) and then present some theorems about the correspondence between parameter rays with preperiodic angles for polynomials and parameter rays with preperiodic addresses for exponentials.

Definition 4.1. We call a dynamic (or parameter) ray pair any couple of dynamic (or parameter) rays landing together whose addresses are preperiodic.

Definition 4.2. Let $\left\{z_{i}\right\}_{i=1 \ldots n}$ be a repelling or parabolic periodic orbit of period $n$ in $\Pi_{c}\left(\Pi_{c}^{D}\right.$ resp.), and

$$
\mathcal{A}_{i}:=\left\{r \in \mathcal{S}\left(\mathbb{S}^{1} \text { resp. }\right): r \text { is periodic and } g_{r}^{c} \text { lands at } z_{i}\right\} .
$$

Then $\mathcal{P}=\left\{\mathcal{A}_{1}, \ldots, \mathcal{A}_{n}\right\}$ is said to be the combinatorial orbit portrait for $\left\{z_{i}\right\}$.

Similarly,

Definition 4.3. Let $\left\{z_{i}\right\}_{i=1 \ldots n}$ be a repelling or parabolic periodic orbit of period $n$ in $\Pi_{c}\left(\Pi_{c}^{D}\right.$ resp.), and $A_{i}:=\left\{g_{r}^{c}: g_{r}^{c}\right.$ lands at $\left.z_{i}\right\}$. Then $P=\left\{A_{1}, \ldots, A_{n}\right\}$ is said to be the orbit portrait for $\left\{z_{i}\right\}$.

Remark 4.4. In a more abstract way, we will speak of a combinatorial orbit portrait without specifying a periodic orbit $\left\{z_{i}\right\}$; such a combinatorial object is not necessarily realized (i.e. occurs for some parameter) as an actual orbit portrait for some polynomial or exponential map.

The next two lemmas are given by Lemma 3.2 in [RS1] for exponentials, and by Lemma 2.3 in $\mathrm{Mi}$ for polynomials.

Lemma 4.5 (Properties of combinatorial orbit portraits). Given a combinatorial orbit portrait $\mathcal{P}=\left\{\mathcal{A}_{1}, \ldots, \mathcal{A}_{n}\right\}$, every $\mathcal{A}_{i} \in \mathcal{P}$ consists of a finite number of periodic addresses (angles resp.), and the shift map sends $\mathcal{A}_{i}$ bijectively onto $\mathcal{A}_{i+1}$. All addresses (angles resp.) share the same period qn, where $q$ is a suitable integer.

Lemma 4.6 (Properties of orbit portraits). Given an orbit portrait $P=\left\{A_{1}, \ldots\right.$, $\left.A_{n}\right\}$, every $A_{i} \in P$ consists of a finite number of dynamic rays, and $f$ maps $A_{i}$ bijectively onto $A_{i+1}$. All dynamic rays in the portrait are periodic with the same period qn, where $q$ is a suitable integer.

The following theorems will show the relation between which combinatorial orbit portraits are realized for exponential maps and which ones are realized for unicritical polynomials. As there are necessary and sufficient conditions for a combinatorial orbit portrait to be realized for polynomials, this gives unique and sufficient conditions for a combinatorial portrait to be realized for exponentials.

Let us first state a correspondence between Misiurewicz parameters for exponential maps and for unicritical polynomials:

Theorem 4.7 (Misiurewicz addresses for exponentials and polynomials). The $p a-$ rameter rays $G_{s_{1}}, \ldots, G_{s_{q}}$ land together at some exponential Misiurewicz parameter in the exponential parameter plane if and only if for each family of unicritical polynomials of sufficiently high degree $D$ the parameter rays with the same addresses land together at some polynomial Misiurewicz parameter. 
Proof. Let $G_{s_{1}}, \ldots, G_{s_{q}}$ be the parameter rays landing at some Misiurewicz parameter $c_{0}$ in the exponential parameter plane. Then the dynamic rays with the corresponding addresses $g_{s_{1}}, \ldots, g_{s_{q}}$ all have the same itineraries with respect to the dynamical partition induced by $g_{s_{1}}$ in $\Pi_{c_{0}}$, because together with $c_{0}$ they form a connected set whose orbit cannot intersect the boundary of the dynamical partition. The address $s_{1}$ is strictly preperiodic, so it is a sequence over finitely many values, so for polynomials of sufficiently high degree $D$ it represents the $\mathrm{D}$-adic expansion of the angle of some parameter ray. As parameter rays with preperiodic angles are well known to land for unicritical polynomials, there is a Misiurewicz parameter $c_{1}$ depending on $D$ which is the landing point of the corresponding parameter ray.

By Theorem 3.2 the dynamic ray $g_{s_{1}}$ lands at $c_{1}$ in the polynomial dynamical plane for $f_{c_{1}}$.

All the polynomial dynamic rays $g_{s_{2}}, \ldots, g_{s_{q}}$ also have the same itinerary with respect to the partition induced by $g_{s_{1}}$, so by Lemma 3.6, they all land together in the dynamical plane for $f_{c_{1}}$. Then by Theorem 3.2 the corresponding parameter rays land together at $c_{1}$ in the polynomial parameter plane.

We will now define characteristic rays (see Lemma 3.3 in [RS1]). We will state the definitions for exponentials, the corresponding definitions for polynomials can be inferred immediately.

Proposition 4.8. Given an orbit portrait, there exist two rays $g_{s_{1}}, g_{s_{2}}$, called characteristic rays, which together with their common endpoint, separate the singular value from all other rays in the portrait. The connected component of $\mathbb{C} \backslash \overline{\left\{g_{s_{1}} \cup g_{s_{2}}\right\}}$ containing the singular value is called the characteristic sector. We will call $s_{1}, s_{2}$ a pair of characteristic addresses.

Definition 4.9. A characteristic parameter ray pair is a pair of parameter rays $G_{s_{1}}, G_{s_{2}}$ with periodic addresses landing together in the parameter plane. The curve $\overline{G_{s_{1}} \cup G_{s_{2}}}$ separates $\mathbb{C}$ into two connected components; the component which contains the rays of addresses between $s_{1}$ and $s_{2}$ is the wake defined by $G_{s_{1}}, G_{s_{2}}$.

The contents of the following proposition are not explicitly stated in the literature. However, they follow from Proposition 5.4 in [RS1] when $c$ is hyperbolic or parabolic, and can be extended by holomorphic motions to all other parameters similarly as in [R1].

Proposition 4.10 (Correspondence of bifurcations). A parameter $c$ belongs to the wake defined by two parameter rays $G_{s_{1}}, G_{s_{2}}$ if and only if the dynamic rays $g_{s_{1}}, g_{s_{2}}$ land together in the dynamical plane $\Pi_{c}$ and are the characteristic rays for some orbit portrait in $\Pi_{c}$.

Theorem 4.11 (Correspondence of characteristic rays). Given a combinatorial orbit portrait $\mathcal{P}$, there exists an exponential map $f_{\tilde{c}}$ realizing $\mathcal{P}$ if and only if there is a unicritical polynomial $P_{c}^{D}$ realizing $\mathcal{P}$, for some sufficiently high degree $D$. In particular, two sequences in $\mathbb{Z}^{\mathbb{N}}$ are characteristic addresses for an exponential map if and only if they are characteristic angles for some unicritical polynomial of sufficiently high degree $D$.

By the definitions and Proposition 4.10, Theorem 4.11 shows that if two polynomial parameter rays $G_{s_{1}}, G_{s_{2}}$ with periodic angles land together in the parameter space $\Pi_{P}^{D}$ for some $D$, then the exponential parameter rays with the corresponding 
addresses land together in $\Pi_{P}$; on the other side, if the parameter rays $G_{s_{1}}, G_{s_{2}}$ land together in $\Pi_{P}$, they land together in $\Pi_{P}^{D}$ for all sufficiently high degree $D$.

Proof. Let $\mathcal{P}=\left\{\mathcal{A}_{i}\right\}$ be a combinatorial orbit portrait. The inverse of the shift map brings each non-characteristic sector to a sector bounded by rays whose addresses/angles have the same first entry, and the characteristic sector to a sector bounded by rays for whose addresses/angles the first entry differs by one.

So, the fact of being a characteristic sector is encoded in the topological orbit portrait, the second claim follows once we show that every combinatorial portrait is realized in the exponential family if and only if it is realized for a polynomial of sufficiently high degree $D$.

If $\mathcal{P}$ is realized for some polynomial $P_{c}^{D}$, and $s, \tilde{s}$ are its characteristic angles, then $\mathcal{P}$ persists in the whole wake bounded by $G_{s}, G_{\tilde{s}}$, so in particular it is realized for some polynomial Misiurewicz parameter as well.

This Misiurewicz parameter is the landing point of a dynamic ray of angle $s$, inducing a dynamical partition as described in Section 3 . The rays whose angles belong to the same $\mathcal{A}_{i}$ land together, so by Lemma 3.6. they have the same itinerary with respect to this partition; in particular, their angles have the same itineraries under the shift map with respect to the partition induced by $s$ in the combinatorial space $\mathcal{S}_{D}$.

By the combinatorial correspondence between polynomials and exponentials, the sequence representing the angle $s$ in $D$-adic expansion can be seen as a strictly preperiodic address $s$ which identifies a Misiurewicz parameter $\tilde{c}$ in the exponential family by Theorem 3.3 . All the dynamic rays whose addresses belong to $\mathcal{P}$ exist in the dynamical plane of $\tilde{c}$, and by Lemma 3.6 they land together as they have the same itinerary with respect to the partition induced by the ray landing at the Misiurewicz parameter.

If $\mathcal{P}$ is realized for an exponential parameter, it persists in the entire wake by Proposition 4.10, so it is realized for some exponential Misiurewicz parameter. Up to selecting a degree $D$ high enough to ensure the existence of the dynamic rays whose addresses belong to $\mathcal{P}$ and of the dynamic rays landing at the exponential Misiurewicz parameter, the specular reasoning can be used to show that $\mathcal{P}$ is realized for some polynomial in the family $\left\{P_{c}^{D}\right\}$.

\section{Fibers AND RIGIDITY}

One of the main problems in one-dimensional complex dynamics, for families of maps for which it is possible to define a natural finite-dimensional parameter space $\mathcal{F}$, is to show that the set of hyperbolic maps is dense in $\mathcal{F}$. Whenever the set of structurally stable parameters $S(\mathcal{F})$ is dense in the parameter space, like for example for exponential maps and unicritical polynomials (see e.g. [EL for greater generality), to say that hyperbolic maps are dense is equivalent to saying that every component of $S(\mathcal{F})$ is hyperbolic.

For exponential maps and unicritical polynomials, thanks to the combinatorial structure carved by parameter rays, this conjecture can be reformulated in combinatorial terms. We will write our discussion for the exponential family, but it can be worked out in parallel also for unicritical polynomials (see [RS2]).

If there was a non-hyperbolic component, by definition of structural stability all maps in a neighborhood of a parameter in this component would be conjugate, so 
that any two maps in the component would have exactly the same set of combinatorial orbit portraits. By the theory of parabolic bifurcation in [RS1, this means that two parameters in the same non-hyperbolic component cannot be separated by a parameter ray pair, or otherwise one of them would have an additional orbit portrait. This leads to the following definitions:

Definition 5.1. The parameter fiber of a parameter $c_{0}$ is the set of parameters which cannot be separated from $c_{0}$ by some pair of parameter rays with strictly preperiodic addresses landing together at a parabolic or Misiurewicz parameter, or by two parameter rays with periodic addresses landing at the boundary of the same hyperbolic component.

By analogy, the dynamical fiber of a point $c_{0}$ is the set of points which cannot be separated from $c_{0}$ by some pair of preperiodic rays landing together at some preperiodic point.

Definition 5.2. We will say that the dynamical or parameter fiber of a point $c_{0}$ is trivial, if any $c \neq c_{0}$ which does not belong to a ray landing at $c_{0}$ can be separated from $c_{0}$ by a pair of preperiodic dynamic or parameter rays landing together.

We will call any result about triviality of fibers a rigidity result. This comes from the fact that any map $f_{c}$ or $P_{c}^{D}$ whose singular value $c$ does not escape and has trivial fiber cannot be conjugate to any other map in a neighborhood because two maps with different orbit portraits cannot be topologically conjugate.

The next result follows immediately from the previous definitions. (See again RS2 for a slightly different formulation of this discussion.)

Theorem 5.3. If the fiber of every non-hyperbolic, non-escaping parameter is trivial, then every component of the set of structurally stable parameters is hyperbolic.

There are two main points in considering fibers to study density of hyperbolicity. The first one is that exponential parameter rays with periodic addresses are closely related to parameter rays for unicritical polynomials (see Theorem 4.11), so that it is possible to infer results about exponentials using known results about polynomials; the second one, which holds in greater generality, is that fibers are a way to "localize" the global conjecture, and select specific classes of parameters which are easier to study.

Our combinatorial rigidity statement (Theorem 1.1) deals with the class of Misiurewicz parameters described in Section 3. We restate it here for convenience:

Theorem 5.4. Fibers of Misiurewicz parameters in parameter space are trivial, i.e. given any postsingularly finite parameter $c_{0}$, for any other parameter $c$ which does not belong to one of the finitely many parameter rays landing at $c_{0}$, there is a pair of parameter rays with periodic addresses which land together at a parabolic parameter and separate $c$ from $c_{0}$.

\section{Triviality of Misiurewicz fiBers}

In this section we prove Theorem 5.4 for the remainder of the section, let $c_{0}$ be a Misiurewicz parameter. The proof follows the outline of the corresponding result for polynomials (Lemma 7.1 and Theorem 7.3 in [S1]), using Theorem 4.7 to establish a bridge between the combinatorics for polynomials and the combinatorics for exponentials. The following is Lemma 7.1 in [S1]. 
Lemma 6.1. Let $c_{0}$ be a Misiurewicz parameter for a family of unicritical polynomials, and let $G_{s_{1}}, \ldots, G_{s_{q}}$ be the parameter rays landing at $c_{0}$. Then for every $\epsilon>0, i=0, \ldots, q-1$, there exist parameter ray pairs $P_{i}$ with angles $\left(\alpha_{i}, \alpha_{i}^{\prime}\right)$ such that

$$
s_{i}<\alpha_{i}<\alpha_{i}^{\prime}<s_{i+1} \text { and } \operatorname{dist}\left(\alpha_{i}, s_{i}\right), \operatorname{dist}\left(\alpha_{i}^{\prime}, s_{i+1}\right)<\epsilon
$$

when $i=1, \ldots, q-1$, and

$$
\alpha_{0}<s_{1}<s_{q}<\alpha_{0}^{\prime} \text { and } \operatorname{dist}\left(\alpha_{0}, s_{1}\right) \text {, } \operatorname{dist}\left(\alpha_{0}^{\prime}, s_{q}\right)<\epsilon .
$$

We will use Lemma 6.1 to prove the following exponential version.

Proposition 6.2 (Combinatorial approximation of parameter rays). Let $c_{0}$ be a Misiurewicz parameter for the exponential family, and let $G_{s_{1}}, \ldots, G_{s_{q}}$ be the parameter rays landing at $c_{0}$. Then for every $\epsilon>0, i=0, \ldots, q-1$, there exist parameter ray pairs $P_{i}$ with addresses $\left(\alpha_{i}, \alpha_{i}^{\prime}\right)$ such that

$$
s_{i}<\alpha_{i}<\alpha_{i}^{\prime}<s_{i+1} \text { and } \operatorname{dist}\left(\alpha_{i}, s_{i}\right), \operatorname{dist}\left(\alpha_{i}^{\prime}, s_{i+1}\right)<\epsilon
$$

when $i=1, \ldots, q-1$, and

$$
\alpha_{0}<s_{1}<s_{q}<\alpha_{0}^{\prime} \text { and } \operatorname{dist}\left(\alpha_{0}, s_{1}\right) \text {, } \operatorname{dist}\left(\alpha_{0}^{\prime}, s_{q}\right)<\epsilon .
$$

At first sight it might seem that this proposition would solve the problem of triviality of fibers, but the relation between the "combinatorial topology" and the topology on $\mathbb{C}$ is from clear, so we still have to show that the parameter ray pairs which approximate the Misiurewicz rays combinatorially actually converge to them with respect to the standard topology on $\mathbb{C}$ in a neighborhood of $c_{0}$. We will derive this from the propositions which follow.

Proposition 6.3 (Triviality in dynamical plane). Let $c_{0}$ be a Misiurewicz parameter for the exponential family. Then dynamical fibers of the postsingular periodic orbit $\left\{z_{i}\right\}$ are trivial.

Given a parameter $\bar{c}$ (for either unicritical polynomials or exponentials), and a repelling periodic orbit $\left\{z_{i}(\bar{c})\right\}$ with period $M$ and combinatorial orbit portrait $\mathcal{P}$, for any $c$ in a sufficiently small parameter neighborhood $U$ of $\bar{c}$ there are $M$ analytic functions $z_{i}(c)$ such that $\left\{z_{i}(c)\right\}$ is a repelling periodic orbit with period $M$ and orbit portrait $\mathcal{P}$ in the dynamical plane for $c$. We will call the orbit $\left\{z_{i}(c)\right\}$ the analytic continuation of $\left\{z_{i}(\bar{c})\right\}$ (see [Mi], Appendix B). Observe that for a given parameter $c$ the orbit $\left\{z_{i}(c)\right\}$, when it exists, is uniquely defined by its orbit portrait.

Proposition 6.4 (Persistence of dynamical triviality). Let $c_{0}$ be as above. The postsingular periodic orbit $\left\{z_{i}\right\}=\left\{z_{i}\left(c_{0}\right)\right\}$ has a well-defined analytic continuation $\left\{z_{i}(c)\right\}$ for $c$ in a neighborhood of $c_{0}$, such that the dynamical fibers of $\left\{z_{i}(c)\right\}$ in $\Pi_{c}$ are trivial.

Let us now prove the previous propositions and Theorem 5.4

Proof of Proposition 6.2. The core of the proof relies on the correspondence between combinatorial spaces for polynomials and for exponential parameters described in the end of Section 2, when the angles labeling rays for polynomials of degree $D$ are written in $D$-adic expansion as sequences over $D$ symbols, they can be seen as a subset of the exponentially bounded sequences encoding the combinatorics for exponential maps. 
Consider the dynamic rays of addresses $s_{1}, \ldots, s_{q}$ landing at the Misiurewicz parameter $c_{0}$ in $\Pi_{c_{0}}$. As noted in Lemma 3.6, each $g_{s_{i}}$ defines a partition with respect to which dynamic rays which are never mapped to $g_{s_{i}}$ have the same itinerary if and only if they land together in the dynamical plane.

Also, $c_{0}$ is the landing point of the parameter rays $G_{s_{1}}, \ldots, G_{s_{q}}$. As $s_{1}, \ldots, s_{q}$ include only finitely many symbols because they are finitely many strictly preperiodic addresses, we can fix a sufficiently high degree $D$ such that the parameter rays of angles $s_{1}, \ldots, s_{q}$ all exist for unicritical polynomials of degree $D$. Choose one of the addresses $s_{1}, \ldots, s_{q}$, say $s_{1}$. As $s_{1}$ is strictly preperiodic, the polynomial parameter ray $G_{s_{1}}$ lands at some polynomial Misiurewicz parameter $\tilde{c_{0}}$ in the family $\left\{P_{c}^{D}\right\}$.

For $\tilde{c_{0}}$ the dynamic ray of angle $s_{1}$ also lands at the singular value in $\Pi_{\tilde{c_{0}}}^{D}$. All other angles $s_{2}, \ldots, s_{q}$ have the same itinerary as $s_{1}$ with respect to the partition induced by $s_{1}$ because they land together in $\Pi_{c_{0}}$, so by Lemma 3.6 the dynamic rays $g_{s_{1}}, \ldots, g_{s_{q}}$ all land together at $\tilde{c_{0}}$. No other dynamic ray can land together with them, otherwise its angle would be an admissible sequence for exponentials and would have the same itinerary, so the corresponding exponential ray would land together with $g_{s_{1}}, \ldots, g_{s_{q}}$ in the exponential dynamical plane as well.

In the dynamical plane $\Pi_{\tilde{c_{0}}}^{D}$ by Lemma 6.1 we have characteristic dynamic ray pairs approximating each sector arbitrarily close. For any such pair of addresses $\left(\alpha, \alpha^{\prime}\right)$, the two rays in the ray pair have the same itinerary by Lemma 3.6 (with respect to the partition induced by $\left.g_{s_{1}}^{D}\right)$, so the two angles $\left(\alpha, \alpha^{\prime}\right)$ have the same itinerary with respect to the partition of the combinatorial space $\mathcal{S}_{D}$ induced by $s_{1}$. Considered as addresses, $\alpha$ and $\alpha^{\prime}$ have the same itinerary in $\mathcal{S}$, so by Lemma 6.1 the exponential rays of addresses $\left(\alpha, \alpha^{\prime}\right)$ land together in $\Pi_{c_{0}}$ giving the wanted approximating ray pairs in the exponential dynamical plane.

Let us transfer the approximating dynamic ray pairs to the parameter plane for exponential maps. By Proposition 4.10, as the approximating rays are characteristic, the parameter rays with the corresponding addresses land together in the exponential parameter plane giving the wanted approximation for the sector defined by $G_{s_{1}}$ and $G_{s_{q}}$. To approximate the other parameter sectors as well, fix a sector, say the sector between $G_{s_{1}}$ and $G_{s_{2}}$, call it $\widehat{s_{1} s_{2}}$.

Let $V \subset \Pi_{P}$ be a neighborhood of $c_{0}$ such that there is an analytic continuation $\tilde{z}(c)$ of $c_{0}$ which keeps all the rays landing at $c_{0}$, and pick a Misiurewicz parameter $c$ in $V \cap \widehat{s_{1} s_{2}}$. In $\Pi_{c}$ we have the same relative position between $\tilde{z}$ and $c$ as we have in parameter plane between $c_{0}$ and $c$, in the sense that $c$ in $\Pi_{c}$ belongs to the sector defined by the rays of addresses $s_{1}$ and $s_{2}$ : this follows from the fact that rays respect the vertical order induced by their addresses both in dynamical and in parameter plane.

Lemma 6.1 gives characteristic dynamic ray pairs approximating $g_{s_{1}}^{c}$ and $g_{s_{2}}^{c}$ for polynomials (now $g_{s_{1}}^{c}$ and $g_{s_{2}}^{c}$ are landing at the repelling point $\tilde{z}(c)$, not at the singular value $c$ ); the corresponding rays can be obtained in the exponential dynamical plane by the same technique as described above, and they can be transferred to the parameter plane by Proposition 4.10.

Note that this proposition assures that we can separate a Misiurewicz parameter from all other Misiurewicz parameters, and from any parameter which is described combinatorially, for example, parabolic and escaping parameters as well as landing points of parameter rays. 
Remark 6.5. By the correspondence of characteristic parameter ray pairs between polynomials and exponentials as stated in Theorem 4.11, we could have obtained the combinatorial approximation directly in the parameter plane, but we need it also in the dynamical plane in order to prove that dynamical fibers of the postsingular orbit are trivial and to proceed with the topological part of the proof.

Proof of Proposition 6.3. Let $z$ be the first periodic point in the postsingular orbit, and $L$ be a linearizing neighborhood for $z$. Let $k$ be the preperiod of $c_{0}$ and $m$ be the period of the postsingular periodic orbit, as in the definition of Misiurewicz parameters. Taking the $k$ th image of the approximating dynamic ray pairs found in the proof of Proposition 6.2 we obtain dynamic ray pairs which approximate combinatorially the $q$ rays $g_{\sigma^{k}\left(s_{1}\right)}, \ldots, g_{\sigma^{k}\left(s_{q}\right)}$ landing at $z$. We want to show that this combinatorial separation corresponds to an actual separation of all points in $L$ from $z$.

So for each sector defined by the $g_{\sigma^{k}\left(s_{i}\right)}$ consider an approximating dynamic ray pair which enters $L$. Note that such a ray pair must exist: as $J\left(f_{c_{0}}\right)=\overline{I\left(f_{c_{0}}\right)}$ and $J\left(f_{c_{0}}\right)=\mathbb{C}$, any open set contains escaping points, so at least one ray must enter each sector, and once there is a ray inside it can be surrounded by one of the combinatorially approximating ray pairs.

Let $\psi$ be the branch of $f^{-m}$ fixing $z$, and for a given sector call $V$ the closed region enclosed by the boundary of the sector, the boundary of $L$ and one of the approximating ray pairs entering that sector. As $V$ does not contain any postsingular point except for $z$, which is fixed by $\psi$, all iterates $\psi^{n}$ are well defined, and because we are in $L$ we have that $\psi^{n}$ tends to $\{z\}$ uniformly on $V$ as $n \rightarrow \infty$.

Proof of Proposition 6.4. Let $\left\{p_{i}\right\}_{i=1 \ldots q}$ be the landing points of the ray pairs which enter the linearizing neighborhood in the proof of Proposition 6.3 then we can find a parameter neighborhood $V$ of $c_{0}$ in which we can continue analytically both the $p_{i}$ 's and the postsingular periodic orbit $\left\{z_{i}\right\}$ with the same rays landing at them.

Up to shrinking $V$, we can also assume that the rays enter the new linearizing neighborhood, and by contraction under the branch of $f^{-m}$ fixing $\left\{z_{i}(c)\right\}$, the neighborhoods between the approximating rays and the actual rays landing at $z_{i}(c)$ shrink to points as in the previous proposition.

Proof of Theorem 5.4. Let us find a parameter neighborhood V of $c_{0}$ so that every $c \in V$ can be separated from $c_{0}$ by a parameter ray pair.

Note that it is enough to separate from $c_{0}$ any parameter $c$ in the bifurcation locus, as rays cannot cross non-hyperbolic components.

We use Propositions 6.3 and 6.4 to show that the combinatorially approximating ray pairs found in Proposition 6.2 converge to the rays landing at $c_{0}$ in the complex plane, so that the domains which we can separate combinatorially actually fill $V \backslash \bigcup G_{s_{i}}$.

As we did before in the dynamical plane, let us distinguish the cases in which the parameter $c$ that we want to separate from $c_{0}$ is in the external sector which contains $-\infty$ (the one bounded by $G_{s_{1}}$ and $G_{s_{q}}$ ) and the case in which $c$ belongs to one of the other sectors.

If $c$ belongs to the external sector, consider the dynamical plane for $c_{0}$, and separate $c$ from $c_{0}$ there by a strictly preperiodic dynamic ray pair, as from Proposition 6.3. follows directly such that the dynamical fiber of $c_{0}$ is trivial. Now it is 
possible to separate this strictly preperiodic ray pair by one of the approximating characteristic parameter ray pairs found in Proposition 6.2 and then transfer this characteristic parameter ray pair into the parameter plane by Proposition 4.10 . Note that $c$ in general does not have a ray landing at it. However, the parameter ray pair and $c$ keep the same relative position in the parameter plane that had $c$ and the corresponding dynamic rays in $\Pi_{c_{0}}$ by Proposition 4.10 .

If $c$ belongs to one of the internal sectors, say $\widehat{s_{1} s_{2}}$, and also belongs to the neighborhood $V$ as in Proposition 6.4 then consider the dynamical plane $\Pi_{c}$. There, $c$ belongs to the corresponding dynamical sector $\widehat{s_{1} s_{2}}$ defined at the analytic continuation $\tilde{z}(c)$. The dynamical fiber of $\tilde{z}$ is trivial by Proposition 6.4, so we can separate $c$ and $\tilde{z}(c)$ by some periodic ray pair $\left(\alpha, \alpha^{\prime}\right)$. This ray pair is persistent over a parameter neighborhood $U$ of $c$. This means that, for parameters in $U$, in the dynamical plane the singular value will be inside the sector bounded by the dynamic rays $\left(\alpha, \alpha^{\prime}\right)$. In particular, by vertical order, escaping parameters in this neighborhood lie on a dynamic ray of address between $\alpha$ and $\alpha^{\prime}$ in the dynamical plane, so they lie on a parameter ray whose address is between $\alpha$ and $\alpha^{\prime}$. By the combinatorial approximation given by Proposition 6.2, such a parameter is separated from $c_{0}$ by any of the ray pairs whose addresses are closer to $s_{1}$ and $s_{2}$ than $\alpha$ and $\alpha^{\prime}$. This means that we can separate all those escaping parameters simultaneously from $c_{0}$ using the same ray pair $\left(\beta, \beta^{\prime}\right)$. By density of escaping points in the bifurcation locus, we can approximate $c$ by escaping parameters, so the ray pair $\left(\beta, \beta^{\prime}\right)$ also separates $c_{0}$ from $c$ unless $c$ lies on $\beta$ or $\beta^{\prime}$, in which case it has a well-defined address and can be separated from $c_{0}$ by any ray pair closer than $\beta$ or $\beta^{\prime}$.

\section{ACKNOWLEDGMENTS}

Many thanks are due to Mikhail Lyubich and Dierk Schleicher for suggesting this problem. Also, discussions with Lasse Rempe, Dierk Schleicher and especially Mikhail Lyubich have been essential in developing and polishing the ideas presented in this paper.

Thanks are also due to the referee for the care devoted to the reports, and for greatly improving the exposition.

The pictures have been drawn with the program It written by Christian Mannes.

\section{REFERENCES}

[BR] Noël Baker, Phil J. Rippon, Iteration of exponential functions, Ann. Acad. Sci. Fenn. Ser. A I Math. 9 (1984), 4-77. MR752391 (86d:58065)

[Be] Walter Bergweiler, Iteration of meromorphic functions, Bull. Amer. Math. Soc. (N.S.) 29 (1993), no. 2, 151-188. MR1216719 (94c:30033)

[B et al.] Clara Bodelon, Robert L. Devaney, Lisa R. Goldberg, Michael Hayes, John H. Hubbard, Gareth Roberts, Dynamical convergence of polynomials to the exponential, J. Differ. Equations Appl. 6 (2000), no. 3, 275-307. MR.1785056 (2001f:37055)

[CG] Lennart Carleson, Theodore W. Gamelin, Complex Dynamics, Springer (1993). MR:1230383 (94h:30033)

[DK] Robert L. Devaney, Michael Krych, Dynamics of Exp(z), Ergodic Theory Dynam. Systems 4 (1984), no. 1, 35-52. MR758892 (86b:58069)

[DH] Adrien Douady, John H. Hubbard, Étude dynamique des polynômes complexes, Prépublications mathématiques d'Orsay (1984/1985), no. 2/4.

[EL] Alexandre Eremenko, Mikhail Lyubich, Dynamical properties of some classes of entire functions, Ann. Inst. Fourier (Grenoble) 42 (1992), no. 4, 989-1020. MR.1196102 (93k:30034) 
[FRS] Markus Förster, Lasse Rempe, Dierk Schleicher, Classification of escaping exponential maps, Proc. Amer. Math. Soc. 136 (2008), no. 2, 651-663. MR2358507 (2008j:37100)

[FS] Markus Förster, Dierk Schleicher, Parameter rays in the space of exponential maps, Ergodic Theory Dynam. Systems 29 (2009), no. 2, 515-544. MR2486782 (2010b:37125)

[GM] Lisa Goldberg, John Milnor, Fixed points of polynomial maps. Part II. Fixed point portraits, Ann. Sci. École Norm. Sup. (4) 26 (1993), no. 1, 51-98. MR1209913 (95d:58107)

[HSS] John H. Hubbard, Dierk Schleicher, Mitsuhiro Shishikura, Exponential Thurston maps and limits of quadratic differentials, J. Amer. Math. Soc. 22 (2009), no. 1, 77-117. MR 2449055 (2010c:37100)

[LSV] Bastian Laubner, Dierk Schleicher, Vlad Vicol, A combinatorial classification of postsingularly finite complex exponential maps, Discrete Contin. Dyn. Syst. 22 (2008), no. 3, 663-682. MR2429858 (2009f:37053)

[McM] Curtis McMullen, Complex Dynamics and Renormalization, Annals of Mathematics Studies (1994), Princeton University Press. MR1312365 (96b:58097)

[Mi1] John Milnor, Periodic orbits, external rays and the Mandelbrot set: an expository account, Astérisques 261 (2000), xiii, 277-333. MR1755445 (2002e:37067)

[Mi] John Milnor, Dynamics in one complex variable, Annals of Mathematics Studies (2006), Princeton University Press. MR2193309 (2006g:37070)

[PR] Carsten L. Petersen, Gustav Ryd, Convergence of rational rays in parameter spaces, in: Tan Lei et al., The Mandelbrot Set: Theme and Variations, Cambridge University Press (2000). MR1765088 (2001f:37057)

[R0] Lasse Rempe, Dynamics of exponential maps, Doctoral Thesis, Christian-AlbrechtsUniversität Kiel (2003), http://e-diss.uni-kiel.de/diss781/.

[R1] Lasse Rempe, A landing theorem for periodic rays of exponential maps, Proc. Amer. Math. Soc. 134 (2006), no. 9, 2639-2648. MR.2213743 (2008d:37073)

[R2] Lasse Rempe, Topological dynamics of exponential maps, Ergodic Theory Dynam. Systems 26 (2006), no. 6, 1939-1975. MR2279273 (2008c:37069)

[RS1] Lasse Rempe, Dierk Schleicher, Combinatorics of bifurcations in exponential parameter space, in: Phil Rippon, Gwyneth Stallard, Transcendental Dynamics and Complex Analysis, London Mathematical Society Lecture Note Series 348 Cambridge University Press (2008), 317-370; arXiv:math/0408011 [math.DS]. MR2458808 (2010e:37059)

[RS2] Lasse Rempe, Dierk Schleicher, Bifurcation loci of exponential maps and quadratic polynomials: local connectivity, triviality of fibers and density of hyperbolicity, in: Mikhail Lyubich et al., Fields Institute Communications Volume 53: Holomorphic dynamics and renormalization. A volume in honor of John Milnor's 75th birthday (2008), 177-196; arXiv:0805.1658 [math.DS]. MR 2477423 (2010b:37133)

[RvS] Lasse Rempe, Sebastian van Strien, Density of hyperbolicity for classes of real transcendental entire functions and circle maps, arXiv:1005.4627v2 [math.DS].

[S0] Dierk Schleicher, On the dynamics of iterated exponential maps, Habilitation Thesis, TU Munchen (1999).

[S1] Dierk Schleicher, On fibers and local connectivity of Mandelbrot and Multibrot sets, Fractal geometry and applications: a jubilee of Benoit Mandelbrot. Part 1, 477-517, Proc. Sympos. Pure Math., 72, Part 1, Amer. Math. Soc., Providence, RI (2004). MR 2112117 (2006b:37088)

[SZ1] Dierk Schleicher, Johannes Zimmer, Escaping points of exponential maps, J. London Math. Soc. (2) 67 (2003), no. 2, 380-400. MR1956142 (2003k:37067)

[SZ2] Dierk Schleicher, Johannes Zimmer, Periodic Points and dynamic rays of exponential maps, Ann. Acad. Sci. Fenn. Math. 28 (2003), no. 2, 327-354. MR1996442 (2004e:37068)

Department of Mathematics, Stony Brook University, Building 5-116, Stony Brook, NEW YORK 11794 